\title{
Morfodinámica y evolución reciente de playa Tunquén, Chile central
}

\author{
Morphodynamics and recent evolution in Tunquen beach, central Chile \\ Carolina Martínez ${ }^{1}$ y Sergio Salinas² \\ ${ }^{1}$ Depto. Geografía, Facultad de Arquitectura, Urbanismo y Geografía, Universidad de Concepción, \\ Casilla 160-C, Concepción, Chile \\ ${ }^{2}$ Escuela de Ciencias del Mar, Facultad de Recursos Naturales. P. Universidad Católica de Valparaíso, \\ Av. Altamirano 1480, Valparaíso, Chile \\ carolmartinez@udec.cl
}

\begin{abstract}
The morphodynamic of Tunquen beach, distal zone of Algarrobo headland bay, central Chile, and its relationship with both, the relative shoreline changes in the mid-term scale and pocket beach conditions, are presented. Tunquen is an intermediate high-energy beach, with transverse bar and rip. A rhythmic topography produced by equidistant megacusps is associated to swash circulations patterns (horn divergent). Sediments correspond to a well classified medium to coarse sand. During the last 45 years, the relative position of the shoreline has shown small variability $( \pm 50 \mathrm{~m})$. This embayment presents stable conditions associated to the shoreline changes and the determined beach type.
\end{abstract}

Key words: Pocket beach, beach cusp, rhythmic morphology, shoreline

\section{Introducción}

La teoría sobre estados morfodinámicos de ambientes costeros de Wright \& Short (1984) se aplica a Tunquén, una playa encajada por promontorios rocosos localizada en la zona distal de la ensenada de Algarrobo en Chile central (Fig. 1). Esta playa se ubica en un contexto geológico de margen continental activo y condiciones micromareales, siendo afectada por un clima de tipo mediterráneo. El principal rasgo morfodinámico observado es la presencia de megacúspides de playa que generan una topografía rítmica asociada a corrientes de retorno. Aunque las cúspides de playa son formas comunes en playas chilenas, mucho se desconoce sobre sus características, relaciones dinámicas con factores geográficos e hidrodinámicos, así como su relación con la estabilidad de la línea litoral. Al respecto, estudios previos han indicado para esta playa, valores importantes de retroceso de la línea litoral de hasta -220 m (ArayaVergara 1985), comportamiento que debería relacionarse con los estados morfodinámicos históricos de la playa o reflejarlo en su tipo de playa. Con el propósito de explorar
Resumen.- Se presentan los estados morfodinámicos de playa Tunquén, zona distal de la ensenada de Algarrobo, Chile central y su relación con cambios en la posición relativa de la línea litoral para escalas temporales de mediano término y sobre condiciones de playa encajada entre promontorios rocosos. Tunquén es un tipo de playa intermedio de alta energía, de barra y canal transversal. Una topografía rítmica con megacúspides de playa equidistantes y estables se asocia a un patrón de circulación de la zona de lavado y retrolavado (swash) que se clasifica como de flujo divergente. Los sedimentos corresponden a arenas medianas a gruesas bien clasificadas. La posición relativa de la línea litoral para los últimos 45 años ha registrado escasa variabilidad ( $\pm 50 \mathrm{~m}$ ). Esta playa presenta equilibrio estable respecto a los cambios de la línea litoral y al tipo de playa encontrado.

Palabras clave: Playa encajada, cúspides de playa, topografía rítmica, línea litoral

estas relaciones, se establecieron como objetivos de este trabajo describir los estados morfodinámicos típicos de playa Tunquén y determinar las variaciones espaciotemporales asociadas a la línea litoral de la playa, a través de un análisis multitemporal de fotografías aéreas para los últimos 40 años y con apoyo de datos de terreno. La necesidad de sistematizar el conocimiento sobre aspectos morfodinámicos de la costa chilena y su evolución para diferentes escalas temporales, se fundamenta especialmente en la tendencia actual a la concentración de actividades económicas y centros poblados que requieren ser orientados sobre la base del conocimiento científico.

En Chile, el conocimiento sobre evolución costera y morfodinámica de playas se ha desarrollado por separado y de manera poco sistemática, aunque un esfuerzo importante se ha realizado en morfodinámica aplicada a ecología de playas arenosas (McLachlan \& Jaramillo 1995, Jaramillo et al. 1999). Araya-Vergara (1996) analiza el comportamiento morfodinámico y las respuestas morfológicas del sistema playa-duna en Chanco y Arauco 


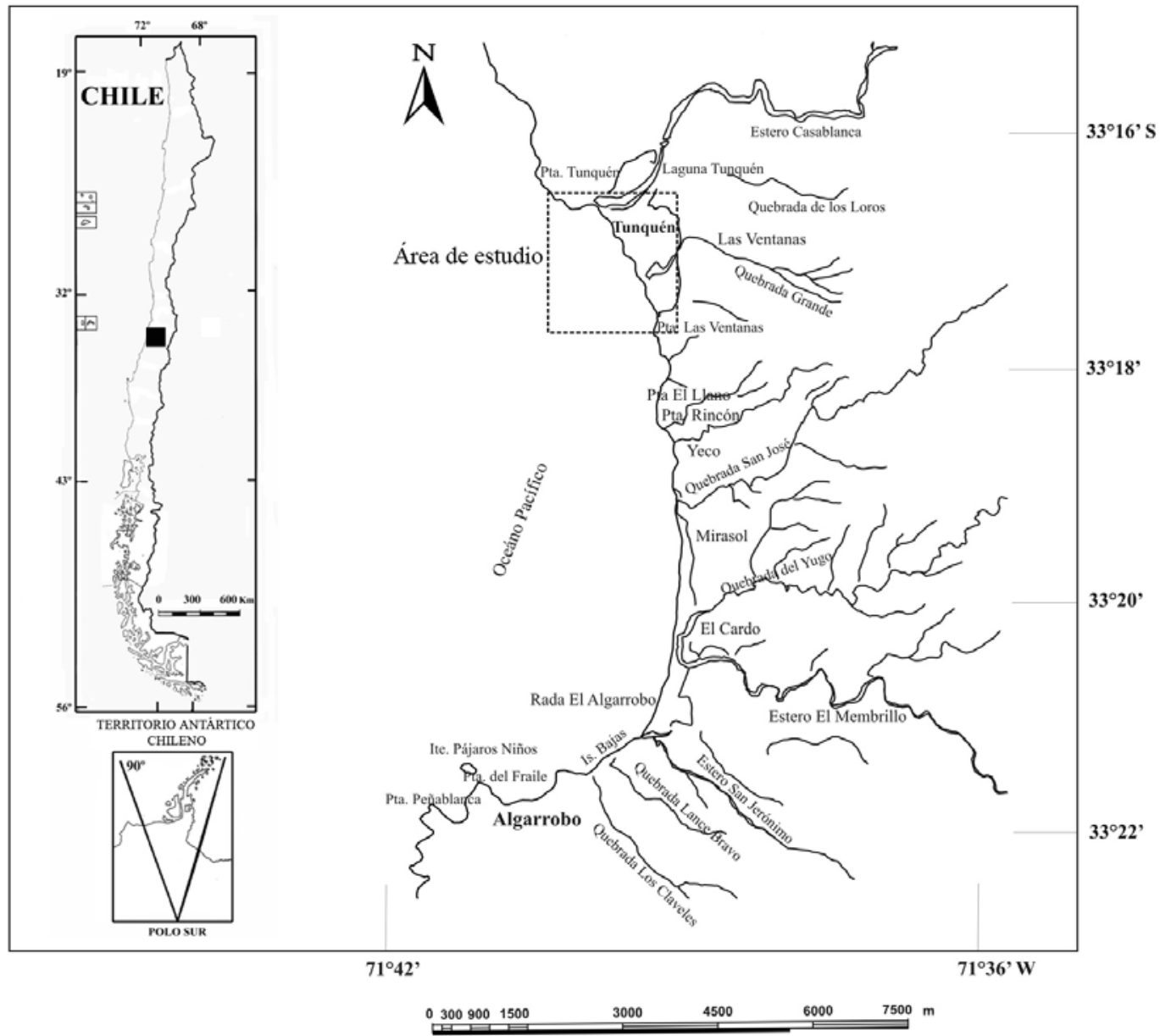

Figura 1

\section{Ubicación de playa Tunquén en la ensenada de Algarrobo}

Location map of Tunquen beach in Algarrobo headland bay

en Chile central sur. Un primer análisis sobre las características morfodinámicas en grandes ensenadas de Chile Central fue realizado por Martínez (2001) diferenciando tipos de playa a través de parámetros adimensionales. Por otro lado, algunas investigaciones para cuantificar los cambios en la posición de la línea litoral en el país han sido realizadas por Araya-Vergara (1985, 1986a) y Martínez (2007). En general, la costa chilena por su extensión, características morfoestructurales y de localización geográfica resulta un área de alto interés para validar propuestas teóricas relacionadas con evolución costera y procesos morfodinámicos.

\section{Material y métodos}

Para determinar los cambios de la posición relativa de la línea litoral en playa Tunquén, se construyó una base cartográfica histórica, sobre la base de fotografías aéreas obtenidas de diferentes vuelos para los últimos 45 años: 1964 (12 marzo), 1967 (07 diciembre), 1977 (23 febrero), 1994 (21 octubre), 2000 (20 marzo) y 2004 (20 febrero). Las fotografías aéreas fueron escaneadas a alta resolución, georreferenciadas, digitalizadas y llevadas al sistema de coordenadas UTM, en datum geodésico WGS-84. La determinación de la línea litoral, se realizó identificando la línea de pleamar. El proceso de georreferenciación se realizó mediante el programa ERDAS 8.0 en el cual, se efectuó un ajuste polinomial a cada uno de los puntos de control que permitió determinar el error medio cuadrático 


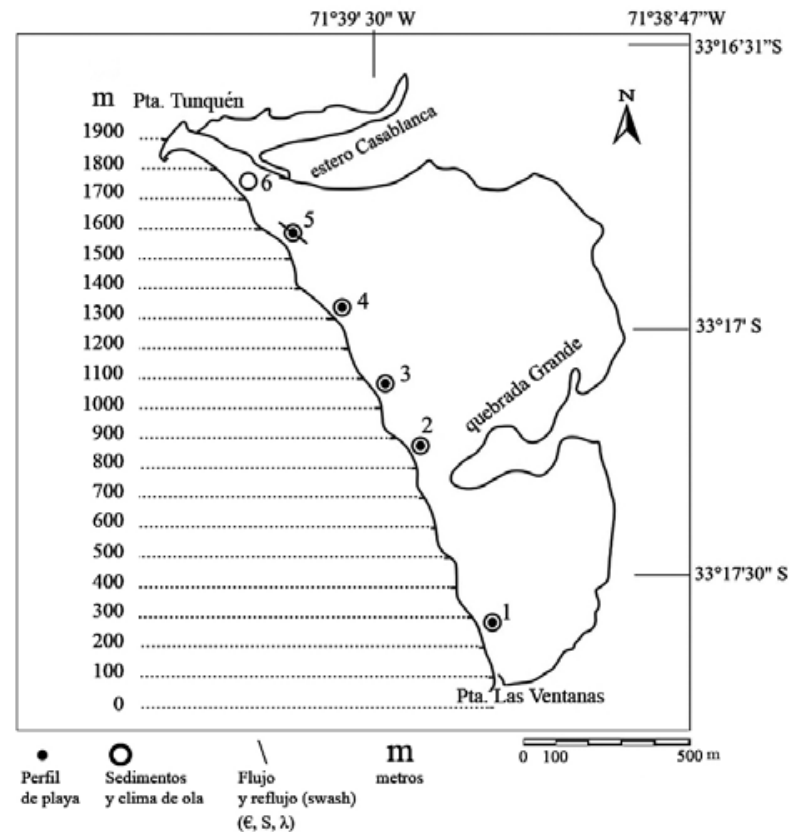

Figura 2

Localización de las estaciones de muestreo y modelo aplicado en la determinación de los cambios en la línea litoral de playa Tunquén

Location of sampling stations and model applied in the detection of shoreline changes of Tunquen beach

(RMS) para cada uno de los modelos. Asimismo, se calculó la resolución del pixel con el objeto de discutir la representatividad de los fotogramas en el terreno y en la determinación de los cambios de la línea litoral (Boak \& Turner 2005).

Se utilizó un criterio de medición sistemático con intervalos de $100 \mathrm{~m}$ a partir del extremo sur del área de estudio (Fig. 2). Se determinaron tres estados de acuerdo a lo indicado por Araya-Vergara (1985 y 1986a): estado de avance (variaciones superiores a $50 \mathrm{~m}$ ); estado estacionario (variaciones entre 0 y $50 \mathrm{~m}$ ); y estado de retroceso (variaciones superiores a $-50 \mathrm{~m}$ ) según el nivel de referencia adoptado. La misma base cartográfica fue utilizada para la determinación del tipo de zona de rompiente de acuerdo a la clasificación de Wright \& Short (1984). Esta clasificación, adaptada por Araya-Vergara (1996) para su trabajo con fotografías aéreas, se utilizó para la identificación del tipo de zona de rompiente.

Para comparar los rasgos morfológicos de la playa a través de la fotointerpretación y con el propósito de generar datos recientes sobre las características morfodinámicas de la playa, se recolectaron datos en una campaña de terreno realizada el 30 de noviembre de 2003. Se establecieron seis estaciones de muestreo para obtener datos de clima de ola, sedimentos y perfiles de playa (Fig. 2). Se realizó el levantamiento topográfico de la línea de playa mediante instrumental topográfico y vinculación geodésica. Este levantamiento fue incluido a la base cartográfica para establecer los cambios en la línea litoral. Los perfiles de playa fueron clasificados de acuerdo a la clasificación morfogenética de Araya-Vergara (1986b) sobre la base de la noción de secuencia.

Las condiciones de la marea se establecieron a partir de la Tabla de Mareas del SHOA(2003) donde la pleamar fue de 1,40 m (06:31 y 19:26 h) y las bajamares de 0,6 m y 0,32 m (0:25 y 13:06 h respectivamente). Las observaciones de clima de ola tales como período y altura de rompiente $(\mathrm{Hb})$ se realizaron para diferentes horas en intervalos de cinco minutos, mientras que la altura de rompiente se obtuvo mediante instrumental topográfico.

En cada estación se obtuvieron muestras de sedimentos superficiales en la zona de rompiente interna, frente de playa ('beachface'), anteduna ('foredune') y duna, obteniéndose un total de 24 muestras de arenas, las cuales fueron analizadas mediante tamizado y clasificadas según la escala Wentworth (1922). Se calcularon los parámetros sedimentológicos de diámetro medio, mediana, asimetría, clasificación y curtosis, de acuerdo a los criterios establecidos por Krumbein \& Pettijohn (1938). Se aplicó el coeficiente de selección So de Trask (1932) para interpretar las condiciones involucradas en el transporte. Los datos de clima de ola y sedimentos 
fueron utilizados para aplicar el parámetro $\Omega$ (Dean 1973) y clasificar el tipo de playa. Se aplicó además el parámetro 'surf similarity' ( $\xi$ ) de Battjes (1974 en Short 1999) para clasificar del tipo de playa, comparar sus resultados con el parámetro anterior $(\Omega)$ y aplicarlo a la determinación de presencia o ausencia de cúspides de acuerdo a lo establecido por Masselink et al. (1997 en Masselink \& Pattiaratchi 1998).

Para caracterizar el estado morfodinámico histórico de la playa, se utilizó la serie de fotografías aéreas con el objeto de identificar el tipo de zona de rompiente y realizar mediciones planimétricas de espaciamiento en las cúspides de playa (longitud de onda) y largo del canal de la corriente de retorno. Se realizaron además observaciones en terreno para las principales cúspides localizadas en la sección norte de la playa (estación $\mathrm{N}^{\mathrm{o}}$ 5). Para la clasificación morfológica de las cúspides de playa se aplicó el parámetro de forma $\Theta(S / \lambda)^{2}$ descrito por Masselink \& Pattiaratchi (1998), que relaciona el patrón de circulación de la zona de lavado y retrolavado ('swash') con la evolución morfológica de la forma. El parámetro $\boldsymbol{E}$ cuantifica la diferencia de la pendiente del frente de playa entre el seno y la cresta de la forma; $\mathrm{S}$ corresponde a la máxima excursión de la zona de lavado (flujo) y $\lambda$ es el espaciamiento de la cúspide. El parámetro $\boldsymbol{\Theta}$ se obtuvo mediante instrumental topográfico. El valor de $\mathrm{S}$ se obtuvo de mediciones realizadas en la zona de lavado para la estación $\mathrm{N}^{0} 5$, en varios momentos de observación.

\section{Resultados}

\section{a) Cambios en la línea litoral}

Se observó en general un estado estacionario en la posición relativa de la línea litoral para el período 1964-
2004, donde solamente el año 1977 sobrepasó el umbral de estabilidad con -98 m registrado en la zona media de la playa. Los años 2000 y 2003 excedieron levemente el nivel estacionario con $-58 \mathrm{~m}$ y $-51 \mathrm{~m}$ respectivamente. Considerando como referencia el año 1994, la playa presentó un leve balance positivo ya que la variación promedio de la línea litoral para este período fue de 1,1 m. Para una escala temporal más reducida (1964-1977), la variación de la línea litoral fue de -22 m. Para otros períodos como 1964-1994; 1964-2000; 1964-2003 las variaciones fueron de -25, -31 m y -12 m respectivamente. En estos casos, la tendencia al retroceso es leve y disminuye a medida que aumenta la escala temporal (Figs. 3 y 4). El RMS calculado para cada año fluctuó entre 0,5 y 1,8 m, con un promedio para la serie histórica de 1,2 m. La representatividad del pixel osciló entre 0,16 m y 1,27 m (Tabla 1).

Tabla 1

\section{Representatividad del pixel y error medio cuadrático (EMC)}

Representativeness of the pixel and root mean square (EMC)

\begin{tabular}{ccc}
\hline Año & Pixel (m) & EMC \\
\hline 1964 & 0,42 & 1,5 \\
1967 & 0,29 & 0,7 \\
1977 & 1,27 & 1,8 \\
1994 & 0,42 & 1,4 \\
2000 & 0,16 & 0,5 \\
2004 & 0,42 & 1,5 \\
\hline
\end{tabular}

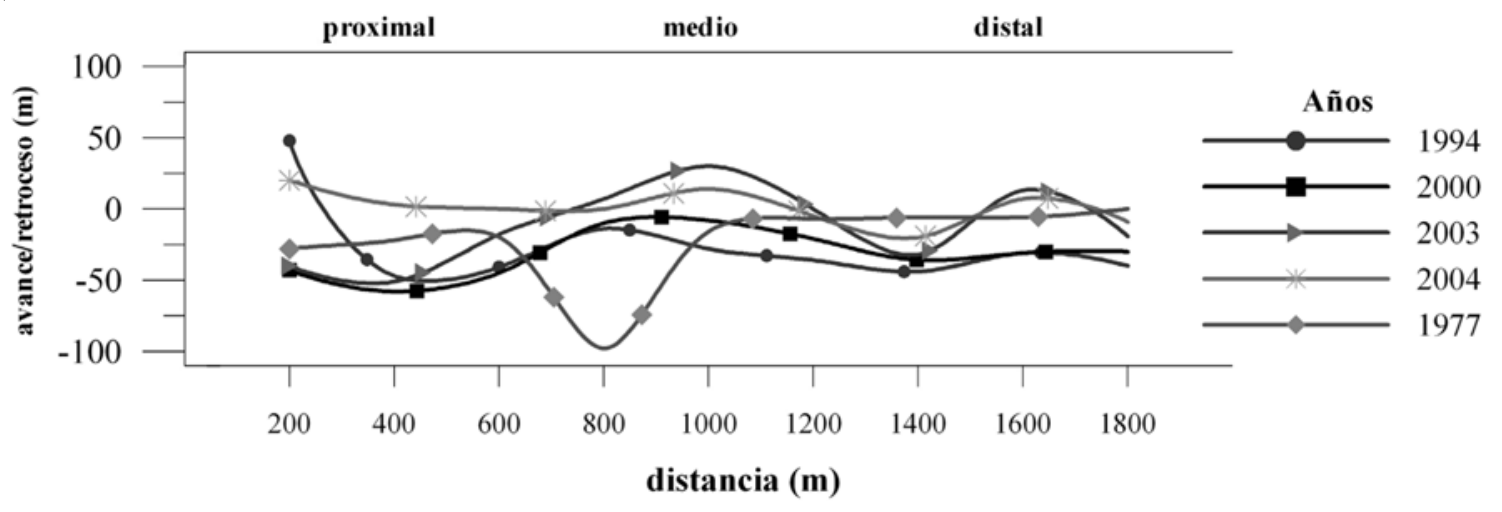

Figura 3

Cambios espacio-temporales de la posición relativa de la línea litoral en playa Tunquén (con referencia al año 1967) 


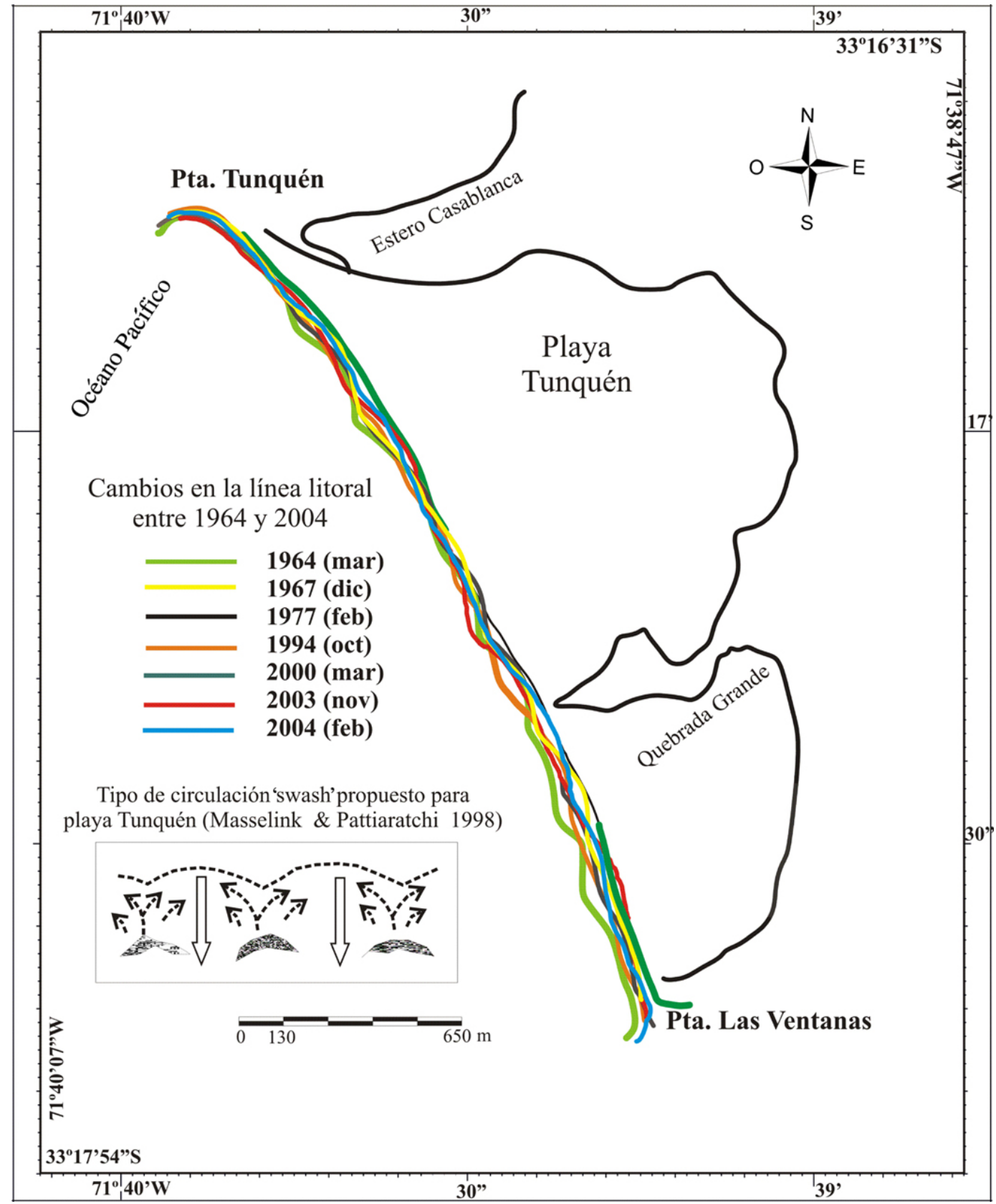

Figura 4

Posición relativa de la línea litoral en playa Tunquén en diferentes años

Shoreline's relative position of Tunquen beach in different years 

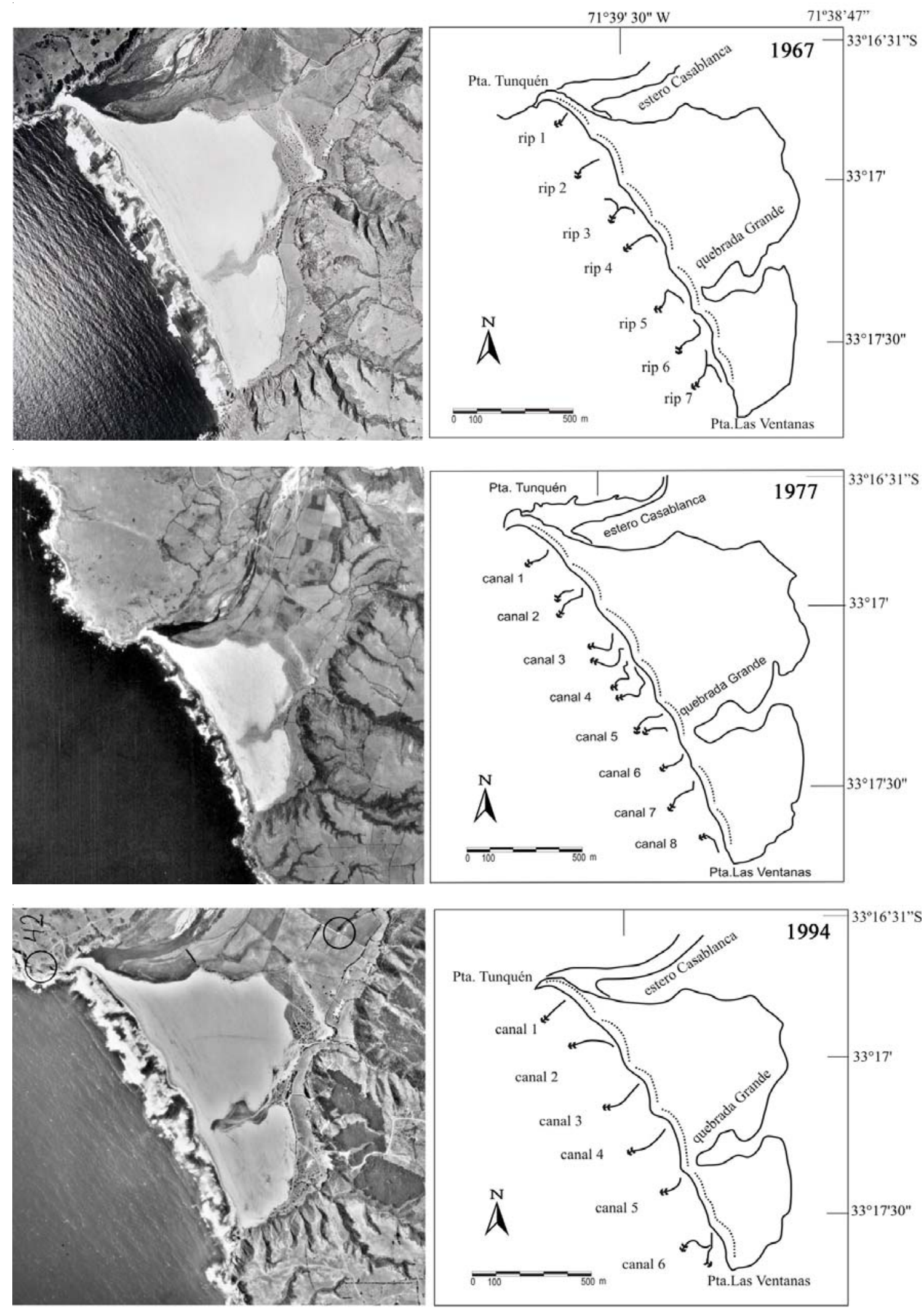
Cont. Fig. 5
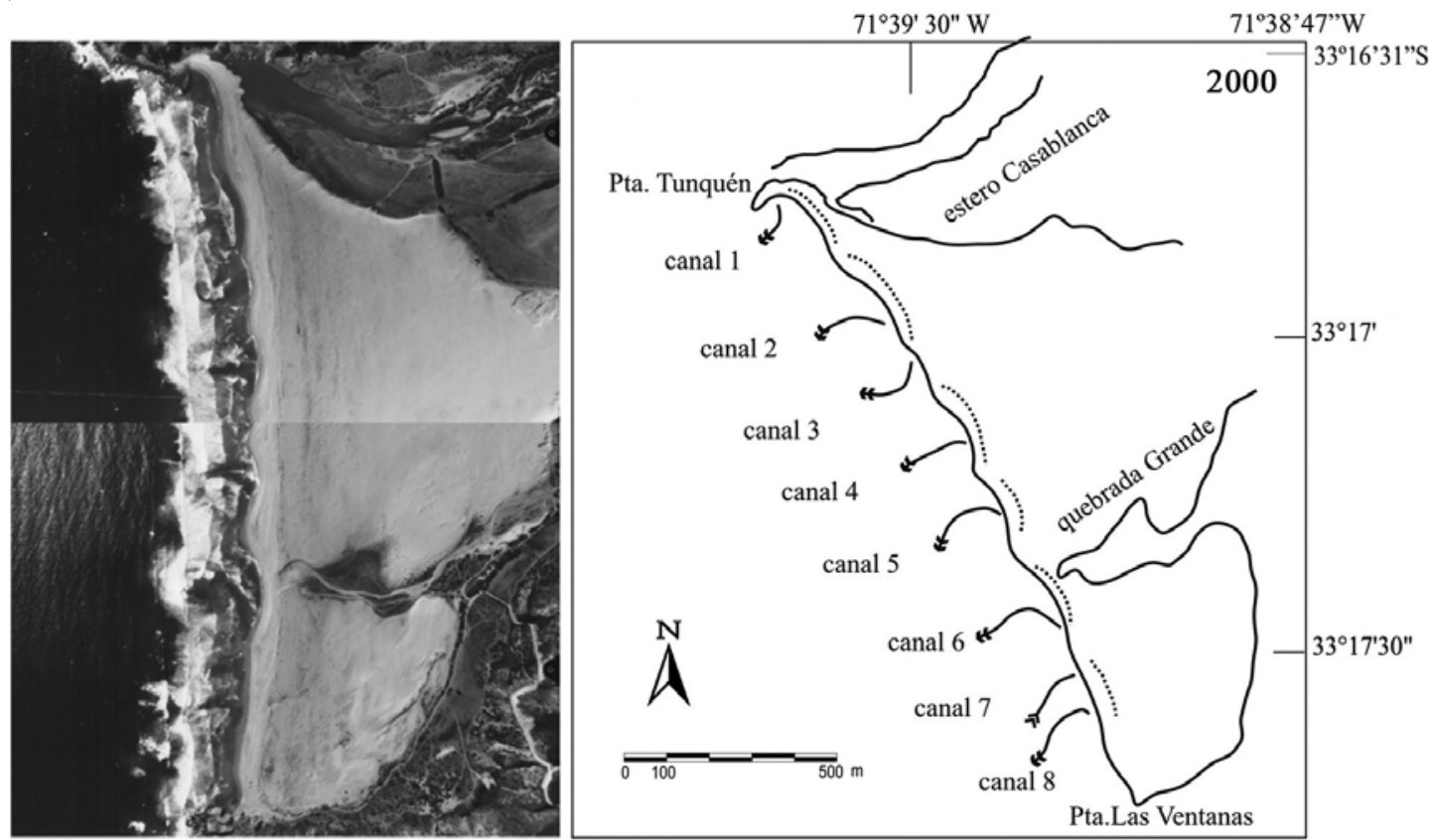

Figura 5

Tipo de zona de rompiente en playa Tunquén en diferentes fechas

Surf zone type at Tunquen beach in different dates

\section{b) Análisis morfodinámico}

\section{Tipo de zona de rompiente}

En la serie histórica de fotografías aéreas se identificó únicamente la categoría de barra y canal transversal, que caracteriza a los tipos de playa con topografías rítmicas (Fig. 5). El ancho promedio de la zona de rompiente fluctuó entre $260 \mathrm{~m}$ en la zona proximal y $218 \mathrm{~m}$ en la zona distal. Los valores más altos de ancho de zona de rompiente correspondieron a 1994. Se presentó una alta correlación entre distancia y ancho de la zona de rompiente $\left(\mathrm{R}^{2}=0,91\right)$ estableciéndose que el ancho de la zona de rompiente aumenta de norte a sur en la playa.

\section{Cúspides de playa y corrientes de retorno}

Se presentaron siete principales cúspides de playa asociados a corrientes de retorno, éstos últimos conectados con la playa a través de canales transversales y también entre sí por surcos laterales. El espaciamiento promedio las cúspides fue de $\lambda=228 \mathrm{~m}$ y el largo promedio de $\mathrm{L}=208 \mathrm{~m}$. Estos se clasificaron como megacúspides de acuerdo con Dolan \& Ferm (1968 en Short 1999).

\section{Condiciones hidrodinámicas}

El clima de ola registró variabilidad en función del momento
Tabla 2

Observaciones del clima de ola (Ts) y (Hb) en playa Tunquén Climate wave observations (Ts) and ( $\mathrm{Hb}$ ) at Tunquen beach

\begin{tabular}{ccc}
\hline Estación / Hora & $\begin{array}{c}\text { Período } \\
(\mathrm{Ts})\end{array}$ & $\begin{array}{c}\text { Altura rompiente }(\mathrm{Hb}) \\
\mathrm{m}\end{array}$ \\
\hline $1(11: 11 \mathrm{~h})$ & 13 & 0,48 \\
$1(11: 20 \mathrm{~h})$ & 15 & 0,47 \\
$2(14: 50 \mathrm{~h})$ & 14 & 0,42 \\
$2(12: 00 \mathrm{~h})$ & 15 & 0,43 \\
$3(15: 20 \mathrm{~h})$ & 13 & 0,34 \\
$3(15: 30 \mathrm{~h})$ & 14 & 0,36 \\
$4(16: 00 \mathrm{~h})$ & 14 & 0,54 \\
$5(18: 00 \mathrm{~h})$ & 13 & 0,43 \\
$6(17: 20 \mathrm{~h})$ & 14 & 0,38 \\
\hline
\end{tabular}

de la observación (Tabla 2). La altura de rompiente (Hb) fluctuó entre 38,8 cm para el perfil $\mathrm{N}^{\circ} 6$ y 48,3 cm en el perfil No 1 . El período de ola (T) fluctuó entre 13 y 15 s. Las mediciones de flujo y reflujo ('swash') realizadas en el perfil $\mathrm{N}^{\mathrm{o}} 5$ registraron una mayor distancia recorrida para el flujo o embestida de la onda que para el reflujo o retroceso en donde se alcanzó valores máximos de $5,4 \mathrm{~m} \mathrm{~s}^{-1}$ al final de las mediciones y en condiciones de pleamar, con un promedio de $2,7 \mathrm{~m} \mathrm{~s}^{-1}$. 


\section{Sedimentos}

La arena mediana fue el rango textural predominante (70,8\%). La arena gruesa comprendió el 25\% y la arena muy gruesa el 4,2\% del total. Se encontró una escasa variabilidad en el rango textural, siendo el diámetro mayor en estaciones de la zona de rompiente. El diámetro medio más bajo se encontró en muestras de duna y anteduna de la zona proximal, mientras que diámetros medios mayores se encontraron en muestras de la zona de rompiente, donde el tamaño promedio (mediana) tiende a aumentar gradualmente desde la zona proximal $(0,406 \mathrm{~mm})$ a la zona distal (1,149 mm) (Fig. 6).

Los sedimentos fueron moderadamente seleccionados para el $87,6 \%$ del total de muestras, mientras que todos los sedimentos de anteduna presentaron selección moderada (Tabla 3). Solo el 8,3\% obtuvo selección pobre (muestras $\mathrm{N}^{\circ} 9$ y 24 ) y el 4,1\% buena selección (muestra $\mathrm{N}^{\circ} 2$ ).

El 54,2\% de las muestras se clasificaron como simétricas (Sk), el 25\% presentó asimetría negativa y un $8,3 \%$ asimetría muy negativa. Se encontraron partículas finas en la cola de la distribución solo en sedimentos de la zona intermareal (muestras $\mathrm{N}^{\circ} 17,18$ y 21) donde el 8,3\% presentó asimetría positiva y el 4,2\% asimetría muy positiva o fina.

Para la curtosis, el 41,6\% de las muestras se clasificó como platicúrticas, principalmente en los sedimentos de la zona de rompiente. El 29,2\% de las muestras fue mesocúrtica. El 16,6\% de las muestras fue leptocúrticas, predominando en sedimentos de duna y anteduna. El 4,2\% resultó muy leptocúrtica (muestras $\mathrm{N}^{\circ} 2$ y 7) y solo el $4,2 \%$ muy platicúrtica (muestra $\mathrm{N}^{\mathrm{o}} 13$ ).

El So de Trask (1932) estableció que el 83,3\% de las muestras presentó buena clasificación, todas correspondieron a sedimentos de frente de playa y anteduna. El 12,5\% de las muestras obtuvo mala clasificación (dos en sedimentos de zona de rompiente y una en duna), mientras que el 4,2\% presentó clasificación moderada.

Se registró una fuerte asociación entre distancia y mediana en las muestras de sedimentos extraídas de la zona de rompiente $\left(R^{2}=0,85\right)$ y frente de playa $\left(R^{2}=0,91\right)$, en menor medida en sedimentos de duna $\left(R^{2}=0,56\right)$, no así en aquellos de anteduna (Fig. 7).

\section{Parámetros morfodinámicos}

Los valores del parámetro $\Omega$ presentaron escasa variabilidad espacial y temporal, fluctuando entre 0,2 y 0,7 en las estaciones extremas de la playa (1 y 6). Estos valores clasifican a la playa como reflectiva (Tabla 4). En general, los valores asociados al clima de ola y velocidad de caída de los sedimentos (Ws) se caracterizaron por bajas fluctuaciones. Sin embargo, el $\Omega$ observado en terreno estableció un $\Omega=3$, lo que determina un tipo de playa de barra y canal transversal. Al igual que $\Omega$, el parámetro ‘surf similarity’ $(\xi)$ clasificó la playa como reflectiva ( $\xi=1.52$ ) (Tabla 5). Al utilizar este parámetro como umbral dinámico para predecir la formación de cúspides, el valor estableció que existen condiciones que favorecen la construcción de cúspides de playa, ya que se cumple $\xi>1,2$. De acuerdo con la relación $\boldsymbol{E}(\mathrm{S} / \lambda)^{2}$ que permite clasificar el tipo de circulación de la zona de lavado y retrolavado en una playa con cúspides o rítmica, la playa Tunquén presentó un patrón de circulación asociado a flujo divergente en el cuerno ('horn divergent') donde $\boldsymbol{\Theta}(\mathrm{S} / \lambda)^{2}=0,10$ lo cual indica un ingreso del flujo a través del cuerno de la cúspide y su salida como reflujo en el centro de la cúspide, favoreciendo su desarrollo.

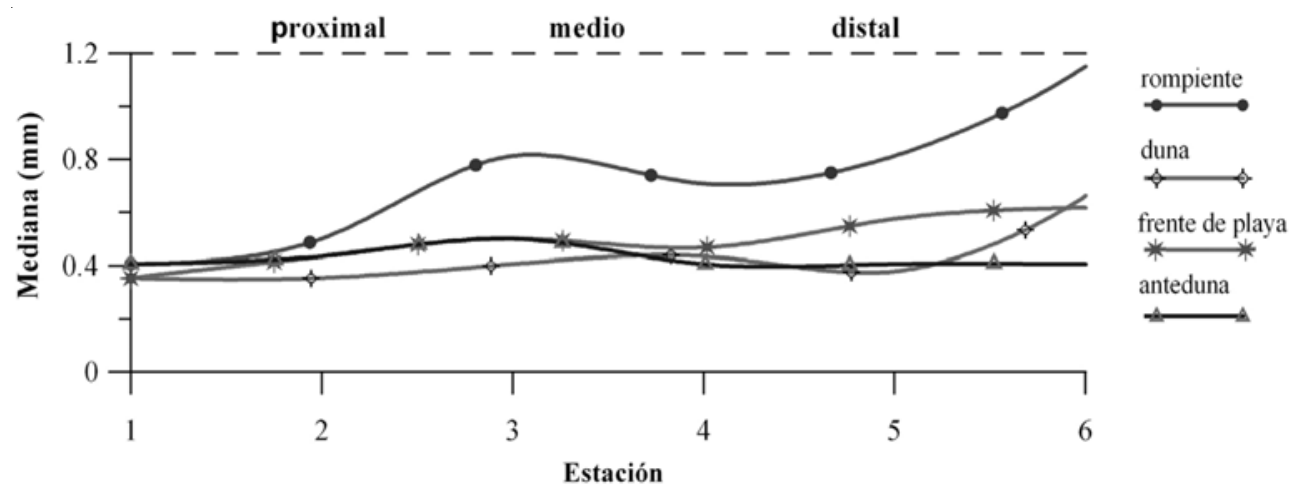

Figura 6

Variaciones en el diámetro promedio de los sedimentos en playa Tunquén

Mean diameter variations of sediments in Tunquen beach 
Tabla 3

Parámetros sedimentológicos en playa Tunquén (30 noviembre, 2003)

Sedimentological parameters at Tunquen beach (November 30, 2003)

\begin{tabular}{|c|c|c|c|c|c|c|c|c|}
\hline $\mathrm{N}^{\circ}$ & Estación & Mediana & Media & Selección & Asimetría & Curtosis & So & Rango textural \\
\hline 1 & P1 rompiente & 0,406 & 0,445 & 0,573 & $-0,278$ & 0,82 & 1,14 & Arena mediana \\
\hline 2 & P1 frente de playa & 0,354 & 0,345 & 0,467 & 0,056 & 1,639 & 1,14 & Arena mediana \\
\hline 3 & P1 anteduna & 0,406 & 0,456 & 0,613 & $-0,324$ & 0,708 & 1,46 & Arena mediana \\
\hline 4 & P1 duna & 0,354 & 0,345 & 0,578 & 0,045 & 1,366 & 1,23 & Arena mediana \\
\hline 5 & P2 rompiente & 0,50 & 0,512 & 0,598 & 0,017 & 0,922 & 1,31 & Arena mediana \\
\hline 6 & P2 frente de playa & 0,435 & 0,477 & 0,623 & $-0,198$ & 0,820 & 1,36 & Arena mediana \\
\hline 7 & $\mathrm{P} 2$ anteduna & 0,435 & 0,50 & 0,553 & $-0,40$ & 2,049 & 1,14 & Arena mediana \\
\hline 8 & P2 duna & 0,354 & 0,354 & 0,764 & $-0,042$ & 0,984 & 1,41 & Arena mediana \\
\hline 9 & P3 rompiente & 0,812 & 0,933 & 1,205 & $-0,033$ & 0,455 & 2,54 & Arena gruesa \\
\hline 10 & P3 frente de playa & 0,50 & 0,50 & 0,623 & 0,056 & 0,820 & 1,36 & Arena mediana \\
\hline 11 & P3 anteduna & 0,50 & 0,50 & 0,638 & $-0,079$ & 0,708 & 1,46 & Arena mediana \\
\hline 12 & P3 duna & 0,406 & 0,456 & 0,784 & $-0,259$ & 1,383 & 1,31 & Arena mediana \\
\hline 13 & P4 rompiente & 0,707 & 0,707 & 0,955 & 0,000 & 0,566 & 2,07 & Arena gruesa \\
\hline 14 & P4 frente de playa & 0,467 & 0,512 & 0,794 & $-0,240$ & 1,066 & 1,41 & Arena mediana \\
\hline 15 & P4 anteduna & 0,406 & 0,425 & 0,748 & $-0,084$ & 0,857 & 1,46 & Arena mediana \\
\hline 16 & P4 duna & 0,435 & 0,477 & 0,985 & $-0,225$ & 1,192 & 1,46 & Arena mediana \\
\hline 17 & P5 rompiente & 0,812 & 0,776 & 0,905 & 0,122 & 0,683 & 1,86 & Arena gruesa \\
\hline 18 & P5 frente de playa & 0,574 & 0,548 & 0,653 & 0,171 & 0,820 & 1,41 & Arena gruesa \\
\hline 19 & P5 anteduna & 0,406 & 0,456 & 0,708 & $-0,212$ & 1,00 & 1,36 & Arena mediana \\
\hline 20 & P5 duna & 0,379 & 0,379 & 0,618 & $-0,024$ & 0,956 & 1,36 & Arena mediana \\
\hline 21 & P6 rompiente & 1,149 & 1,047 & 0,794 & 0,317 & 1,066 & 1,41 & Arena muy gruesa \\
\hline 22 & P6 frente de playa & 0,616 & 0,588 & 0,748 & 0,041 & 0,857 & 1,46 & Arena gruesa \\
\hline 23 & P6 anteduna & 0,406 & 0,406 & 0,729 & $-0,020$ & 1,464 & 1,27 & Arena mediana \\
\hline 24 & P6 duna & 0,660 & 0,660 & 1,352 & 0,012 & 0,928 & 1,93 & Arena gruesa \\
\hline
\end{tabular}

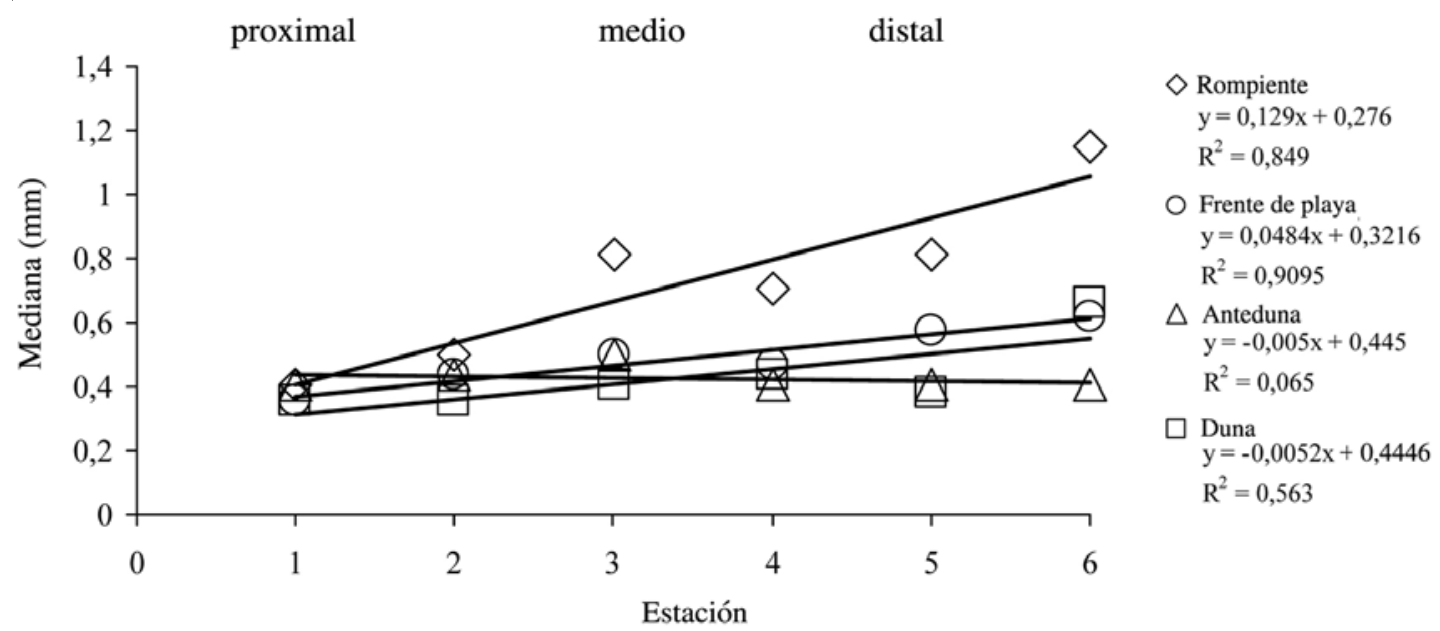

Figura 7

Correlación lineal $\left(R^{2}\right)$ entre distancia en el sistema y diámetro promedio (mediana) en sedimentos de playa Tunquén

Lineal correlation $\left(\mathrm{R}^{2}\right)$ between distance to system and mean diameter (median) of sediments in Tunquen beach 
Tabla 4

Parámetros morfodinámicos de playa Tunquén

Morphodynamics parameters in Tunquen beach

\begin{tabular}{lccccccc}
\hline Estación / Hora & $\begin{array}{c}\mathrm{Hb} \\
(\mathrm{m})\end{array}$ & $\begin{array}{c}\mathrm{T} \\
(\mathrm{s})\end{array}$ & $\begin{array}{c}\mathrm{D}_{50} \\
(\mathrm{~mm})\end{array}$ & $\begin{array}{c}\mathrm{Ws} \\
\left(\mathrm{m} \mathrm{s}^{-1}\right)\end{array}$ & $\begin{array}{c}\Omega \\
\text { calculado }\end{array}$ & $\begin{array}{c}\Omega \\
\text { observado }\end{array}$ & $\begin{array}{c}\text { Tipo playa } \\
\text { calculado }\end{array}$ \\
\hline $1(11: 11 \mathrm{~h})$ & 0,48 & 13 & 0,4 & 0,05 & 0,7 & 3 & Reflectiva \\
$1(11: 20 \mathrm{~h})$ & 0,47 & 15 & 0,4 & 0,05 & 0,6 & 3 & Reflectiva \\
$2(14: 50 \mathrm{~h})$ & 0,42 & 14 & 0,5 & 0,05 & 0,6 & 3 & Reflectiva \\
$2(12: 00 \mathrm{~h})$ & 0,43 & 15 & 0,5 & 0,05 & 0,6 & 3 & Reflectiva \\
$3(15: 20 \mathrm{~h})$ & 0,34 & 13 & 0,8 & 0,09 & 0,3 & 3 & Reflectiva \\
$3(15: 30 \mathrm{~h})$ & 0,36 & 14 & 0,8 & 0,09 & 0,3 & 3 & Reflectiva \\
$4(16: 00 \mathrm{~h})$ & 0,54 & 14 & 0,7 & 0,07 & 0,6 & 3 & Reflectiva \\
$5(18: 00 \mathrm{~h})$ & 0,43 & 13 & 0,8 & 0,09 & 0,4 & 3 & Reflectiva \\
$6(17: 20 \mathrm{~h})$ & 0,38 & 14 & 1,1 & 0,15 & 0,2 & 3 & Reflectiva \\
\hline
\end{tabular}

Tabla 5

Síntesis morfodinámica de playa Tunquén

Morphodynamics synthesis of Tunquen beach

\begin{tabular}{cccccccc}
\hline Lo & $\mathrm{S}$ & $\Lambda$ & $\epsilon$ & $\epsilon(\mathrm{S} / \lambda)^{2}$ & $\tan \beta$ & $\mathrm{Hb} \mathrm{m}$ & $\xi$ \\
\hline 263,771 & 2,80 & 321 & 0,143 & 0,10 & 0,061 & 0,43 & 1,52 \\
\hline
\end{tabular}

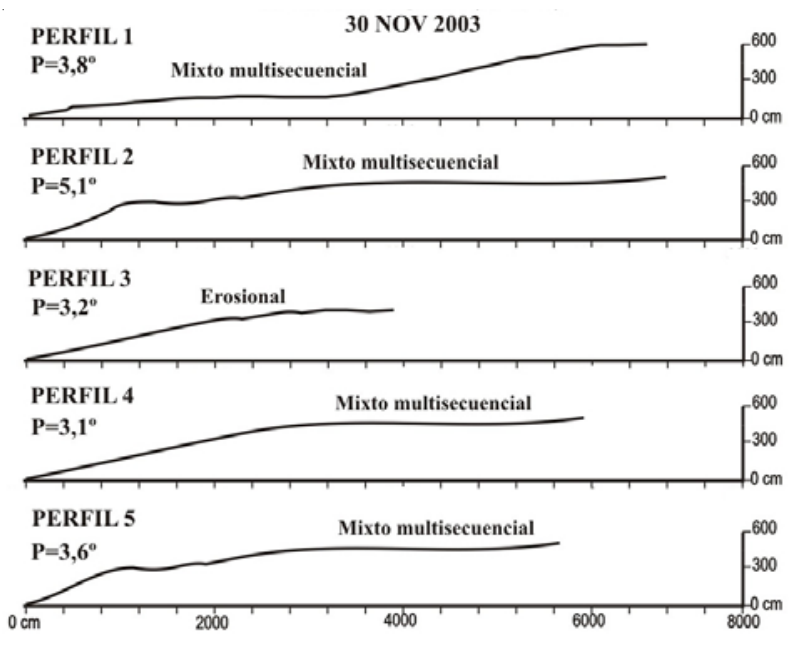

Figura 8

Perfiles de playa en Tunquén (estaciones 1-5). $\mathbf{P}=$ pendiente del perfil

Beach profiles in Tunquen (stations 1-5). $\mathrm{P}$ = slope

\section{Perfiles de playa}

Los perfiles de playa realizados en Tunquén correspondieron en su mayoría a secuencias erosivas con una sección cóncava inicial de pendiente fuerte ocupando el frente de playa hasta el límite de las cúspides, máximo alcance de la onda de lavado y retrolavado (Fig. 8). El límite superior de las cúspides se alcanzó en promedio a los $15 \mathrm{~m}$ desde el inicio de la onda de lavado, con pendientes entre $3,1^{\circ}$ y $5,1^{\circ}$. A lo largo de la playa se encontraron secuencias alternantes de sección cóncava en el centro de la cúspide y convexa en los cuernos de éstas. De acuerdo con la clasificación morfogenética de Araya-Vergara (1986), los perfiles se clasificaron como mixtos multisecuenciales debido a las secuencias alternantes erosivas y de acreción. Solo el perfil $\mathrm{N}^{0} 3$ se clasificó como erosional aunque en este caso solo pudo observarse la primera secuencia del perfil y no el perfil completo.

\section{Discusión}

Las variaciones espacio-temporales de la línea litoral en playa Tunquén para el período 1964-2004 registraron un 
promedio de $1,1 \mathrm{~m}$ con un error medio $\pm 1,2 \mathrm{~m}$ lo cual establece condiciones de fuerte estabilidad en la línea litoral. Si se considera el grado de representación de la serie histórica, el 86\% de los fotogramas utilizados incluyó escalas entre 1:8.000 y 1:20.000 (1964, 1967, 1994, 2000 y 2004), consideradas adecuadas para su uso ya que en el primer caso la representatividad del pixel en relación al terreno fue de $0,16 \mathrm{~m}$ y para el segundo de $0,42 \mathrm{~m}$ con RMS inferiores a 1,8 m. Solo el fotograma del año 1977 presentó una escala 1:60.000 involucrando una representatividad de 1,27 m y un RMS máximo de 1,8 m. Esto último puede explicar la ligera tendencia de la línea litoral a acentuar los cambios en planta respecto al resto de los años en donde se pudo observar un comportamiento relativamente similar. Desde el punto de vista estacional, el 71\% de las fotografías aéreas de la serie histórica correspondió a condiciones típicas de verano y el $29 \%$ restante, a primavera. La falta de datos representativos de la componente invernal hace que la interpretación espacio-temporal de los resultados deba limitarse a un comportamiento estacional típico de verano. Aún así, la escala temporal es capaz de reflejar una tendencia que podría tener un comportamiento variable para escalas temporales reducidas (interanual o estacional), si se incorpora en el análisis los efectos de las tormentas invernales o de marejadas en verano, de las cuales existen escasos estudios (Fonseca 1985).

Los resultados sobre cambios asociados a la línea litoral de playa Tunquén no son concordantes con los estudios previos que han establecido una fuerte tendencia al retroceso de -200 m para el período 1955-1978 y de hasta -263 m para períodos aún más cortos 1955-1963 (Araya-Vergara 1985). Esto último podría estar relacionado con las técnicas o métodos utilizados para determinar los cambios.

La escasa variabilidad en la posición relativa de la línea litoral de playa Tunquén, con valores que caracterizan el estado estacionario, parece seguir la tendencia descrita por Martínez (2007) para grandes ensenadas de Chile central entre 1967 y 2004, determinando estado estable para las bahías de Concón y Algarrobo. Esta tendencia se encontró además asociada a tipos de playa intermedios o reflectivos, estos últimos considerados los más estables de acuerdo a la clasificación de Wrigth \& Short (1984). En playa Tunquén se determinó únicamente el tipo de playa de barra y canal transversal, conteniendo seis cúspides de playa permanentes en toda la serie histórica. Sin embargo, los parámetros morfodinámicos aplicados no reflejaron este tipo de playa ya que tanto $\Omega$ (calculado) y $\xi$ entregaron valores indicativos para el tipo reflectivo. Esto último podría relacionarse con la escasa representatividad temporal de los datos en el uso de los índices morfodinámicos. Tal situación ha sido relatada por varios autores en diferentes partes del mundo. Muehe (1998) analizando playas brasileñas estableció que el parámetro $\Omega$ se aplica bien a playas reflectivas pero no así en intermedias y disipativas. Resultados similares fueron obtenidos por Martínez (2001) al comparar el tipo de playa en grandes ensenadas de Chile central. Gómez-Pujol et al . (2007) utilizaron la clasificación de Wrigth \& Short (1984) en playas de baja energía en la costa de Mallorca, resultados que han sido fuertemente criticados por Jiménez et al. (2008), al compararla con datos obtenidos de cámaras de video y modelamiento numérico, estableciendo la escasa resistencia de $\Omega$ a la validación con otros métodos.

La interpretación morfodinámica en función del uso de parámetros predictivos $(\Omega, \xi$, $\Theta$ ) es uno de los aspectos más discutidos en la literatura actual ya que su determinación es fuertemente dependiente de la disponibilidad de series de tiempo. En el caso de esta investigación, se requiere construir series de tiempo para datos sobre morfodinámica dado que no es posible generalizar sobre estados típicos a partir de una sola campaña de terreno como en este caso, especialmente debido a las tasas de cambio que afectan a las playas, las cuales suelen ser mayores en playas intermedias y disipativas (Short 1987). En este sentido, Wright et al. (1984) encontraron que la predicción sobre el estado morfodinámico en una playa requiere datos referidos a procesos morfológicos de al menos 30 días en playas energéticas y probablemente más sobre playas menos energéticas (Short 1999, 2006). Para la elaboración del modelo morfodinámico de playas, Wrigth \& Short (1984) utilizaron 6,5 series de tiempo en playas australianas, con datos diarios de clima de ola y sedimentos.

Respecto a los rasgos morfológicos asociados al tipo de playa en Tunquén, se estableció que las cúspides de playa asociadas a corrientes de retorno constituyen los elementos más sobresalientes debido a su permanencia en la serie histórica analizada:

a) La formación de cúspides de playa generalmente asociados a presencia de corrientes de retorno, clasificados aquí como megacúspides (Dolan \& Ferm 1968 en Short 1999) o cúspides de topografías rítmicas, típicos de playas intermedias (Short 1999). Una de las hipótesis más aceptadas para explicar la formación de cúspides de playa es la de las ondas atrapadas a la costa, conocidas como ondas de borde fijas ('standing edge wave'), que se verían favorecidas por una incidencia normal a la costa (Holland \& Holman 1996), condición que se presenta en Tunquén. Considerando los datos para Narrabeen Beach, Short (1985) pudo establecer 
relaciones entre $\gamma_{\mathrm{s}}$ y $\Omega$ en donde se obtuvo que los canales de acreción se inician cuando $\Omega<2,5$ mientras que los canales de erosión persisten cuando $\Omega>2,5$. En playa Tunquén, los valores $\Omega$ entre 0,2 y 0,7 encontrados para $\gamma_{\mathrm{s}}=270 \mathrm{~m}$ en promedio establecieron presencia de canales de acreción, situación que refleja las condiciones encontradas, esperándose efectos morfológicos tales como presencia de cordones de anteduna y perfiles de playa mixtos aún presentando una secuencia inicial erosiva como los encontrados aquí.

Por otro lado, Masselink \& Pattiaratchi (1998) han indicado que la destrucción de cúspides ocurre cuando $\xi$ $<1,2$ mientras que su construcción suele ocurrir cuando $\xi$ $>1,2$ bajo tipos de rompiente en voluta o brava. En Tunquén se encontró esta última condición, en donde $\xi=1,52$ por lo tanto se encontraron condiciones que favorecen la construcción de cúspides. Así, el parámetro $\xi$ predijo bien la presencia de las cúspides pero no así el estado morfodinámico de la playa. Finalmente, si se consideran los resultados del parámetro $\mathfrak{E}(\mathrm{S} / \lambda)^{2}=0,10$ y los criterios propuestos por Masselink \& Pattiaratchi (1998), la zona de lavado y retrolavado en playa Tunquén presenta un flujo divergente en el cuerno y reflujo en el centro de la cúspide lo cual favorecería la permanencia de las cúspides de playa.

b) La presencia de corrientes de retorno que se presentan conectadas al centro de las cúspides de playas en playa Tunquén. Suelen ser asociadas a un fenómeno especial de dinámica de fluidos y generalmente se explican a través de la hipótesis de las ondas atrapadas por la costa. Se han reportado en diferentes ambientes y distintas velocidades. Bujalesky (1997) las encontró asociadas a canales de sobrelavado y espaciamiento modal de 90 m en la costa atlántica de Tierra del Fuego (espiga El Páramo), generadas por olas con períodos entre 11 y $17 \mathrm{~s}$. En una playa típica con altura de onda inferior a 1,5 $\mathrm{m}$ al valor máximo puede alcanzar desde $1 \mathrm{~m} \mathrm{~s}^{-1} \mathrm{a} 2 \mathrm{~m} \mathrm{~s}^{-1}$ (Short \& Hogan 1994), mientras que las velocidades esperadas en los megacanales pueden exceder los 2 a 3 $\mathrm{m} \mathrm{s}^{-1}$ (Wright et al. 1980 en Short 1985). En playa Tunquén, el experimento realizado en la estación $\mathrm{N}^{\circ} 5$ estableció velocidades promedio de $2,7 \mathrm{~m} \mathrm{~s}^{-1}$ concordantes con los valores establecidos para megacanales aunque dicho valor no puede ser generalizado por tratarse solo de un único experimento. La presencia de las cúspides en Tunquén podría ser favorecida por un clima de ola en Chile central relativamente estable. Araya-Vergara (1971) y Martínez (2001) para estaciones de marea de Constitución y Valparaíso, coinciden en que las alturas de ola significativa se distribuyen en un $88 \%$ en el rango de los 0,5 a 2,5 m, mientras que el $75 \%$ de las alturas de ola máxima según período se distribuyen entre los 8 y 12 s.
La caracterización de playas de Chile central de Del Canto \& Paskoff (1982), reportó la presencia de cúspides de playa tanto para playa Algarrobo (inmediatamente al sur del área de estudio) y en playa Tunquén. Los rangos texturales promedios encontrados por estos autores para las cúspides de playas fueron de $0,56 \mathrm{~mm}$ en el cuerno y $0,62 \mathrm{~mm}$ en el cúspide, correspondientes a arenas gruesas, condiciones similares a las encontradas en playa Tunquén. Anteriormente, González (1976) determinó para las arenas de playa Tunquén, una mediana de $0,37 \mathrm{~mm}$ coincidente también con el rango predominante encontrado en esta investigación para el $71 \%$ de las muestras analizadas. Del mismo modo, González (1976) reporta valores de $\mathrm{Sk}=1$ y $\mathrm{So}=1,02$ lo cual coincide también con los valores encontrados aquí. Lo anterior indica que en playa Tunquén los procesos asociados al transporte sedimentario han sido estables en el área.

Del análisis morfodinámico y de la posición relativa de la línea litoral realizado, es posible establecer que las variables diagnósticas asociadas al tipo de playa tales como parámetros sedimentológicos (mediana y So), tipo de zona de rompiente (barra y canal transversal) y formas predominantes (cúspides de playa) indican condiciones de estabilidad respecto de los procesos litorales para el período analizado. Lo anterior coincide con las condiciones estables de la línea litoral para los últimos 45 años en playa Tunquén. Estas relaciones deberían ser incluidas en las futuras investigaciones con el objeto de abrir esta línea de trabajo en el país para conocer sus implicancias en la evolución de la costa chilena.

\section{Agradecimientos}

Se agradece el entusiasmo y apoyo en terreno de los entonces alumnos de la Carrera de Geografía Carlos Romero, Pablo Villar y Jacqueline De Rurange, así como de la colaboración técnica del Sr. Luis Flores en el levantamiento topográfico. Se agradece muy especialmente a los evaluadores anónimos que aportaron valiosas sugerencias al manuscrito.

\section{Literatura citada}

Araya-Vergara J. 1971. Determinación preliminar de las características del oleaje en Chile central. Noticiero Mensual Museo Nacional de Historia Natural 15(174): 8-12.

Araya-Vergara J. 1985. Trend analysis of shoreline changes and coastal management in Central Chile (33-34.5 $\left.{ }^{\circ} \mathrm{S}\right)$. En: Actes Excursion-Symposium $\mathrm{N}^{\circ}$ 9: La Côte Atlantique, pp. 99-110. Union Géographique Internationale. Commission sur l'Environnement Côtier, Paris.

Araya-Vergara J. 1986a. Cambios actuales de la línea litoral en Chile central sur. Revista Geográfica de Chile Terra Australis 29: 23-28. 
Araya-Vergara J. 1986b. Toward a classification of beach profiles. Journal of Coastal Research 2(2):159-165.

Araya-Vergara J. 1996. Sistema de interacción oleaje-playa frente a los ergs de Chanco y Arauco, Chile. Gayana Oceanología 4(2): 159-167.

Boak E \& I Turner. 2005. Shoreline definition and detection: a review. Journal of Coastal Research 21(4): 688-703.

Bujalesky G. 1997. Patrón especial y dinámica de canales de sobrelavado de la costa atlántica septentrional de Tierra del Fuego. Revista de la Asociación Geológica Argentina 52(3): 257-274.

Dean R. 1973. Heuristic models of sand transport in the surf zone. Proceedings of the First Australian Coastal Engineering, pp. 208-214. Institute Engineers Australia, Sydney.

Del Canto S \& R Paskoff. 1982. Características y evolución geomorfológica actual de algunas playas de Chile central, entre Valparaíso y San Antonio (V Región). Revista de Geografía Norte Grande 10: 31-45.

Fonseca T. 1985. Observaciones sobre los temporales que afectaron la bahía de Valparaíso en julio de 1984. Revista Geográfica de Valparaíso 16: 31-46.

Gómez-Pujol L, A Orfila, B Cañellas, A Alvarez-Ellacuria, F Méndez, R Medina \& J Tintoré. 2007. Morphodynamic classification of sandy beaches in low energetic marine environment. Marine Geology 242: 235-246.

González I. 1976. Sedimentología litoral de la provincial de Valparaíso, Chile. Primer Congreso Geológico Chileno, 27 agosto, Santiago de Chile, pp. E217-E241.

Holland K \& R Holman. 1996. Field observations of beach cusps and swash motions. Marine Geology 134: 77-93.

Jaramillo E, F Carrasco, P Quijón, M Pino \& E Contreras. 1999. Distribución y estructura comunitaria de la macroinfauna bentónica en la costa del norte de Chile. Revista Chilena de Historia Natural 72: 459-479.

Jiménez J, J Guillén \& A Falqués. 2008. Comment on the article 'Morphodynamic classification of sandy beaches in low energetic marine environment' by Gómez-Pujol L, A Orfila, B Cañellas, A Alvarez-Ellacuria, F Méndez, R Medina \& J Tintoré. Marine Geology 242: 235-246, 2007. Marine Geology 255: 96-101.

Krumbein W \& F Pettijohn. 1938. Manual of sedimentary petrography, 500 pp. Appleton Century Crofts, New York.
Martínez C. 2001. El efecto de ensenada en los procesos litorales de las ensenadas de Valparaíso, Algarrobo y Cartagena, Chile central. Tesis de Magíster en Geografía, Escuela de Postgrado, Universidad de Chile, Santiago, 152 pp.

Martínez C. 2007. Shoreline changes in Concón and Algarrobo bays, Central Chile, using and adjustment model. Investigaciones Marinas 35(2): 99-112.

Masselink G \& C Pattiaratchi. 1998. Morphological evolution of beach cusps and associated swash circulation patterns. Marine Geology 146: 93-113.

McLachlan A \& E Jaramillo. 1995. Zonation of sandy beaches. En: Ansell A, R Gibson \& M Barnes (eds). Oceanography and Marine Biology: an Annual Review 33: 305-335.

Muehe D. 1998. Estado morfodinâmico praial no instante da observação: una alternativa de identificação. Revista Brasileira de Oceanografía 46(2): 157-169.

SHOA. 2003. Tablas de marea de la costa de Chile. Servicio Hidrográfico y Oceanográfico de la Armada de Chile, Publicación 3009: 1-237.

Short A. 1985. Rip current type, spacing and persistence, Narrabeen Beach, Australia. Marine Geology 65: 47-71.

Short A. 1987. A note on the control of beach type and change, with S.E. Australian examples. Journal of Coastal Research 3(3): 387-395.

Short A. 1999. Handbook of beach and shoreface morphodinamics, 379 pp. John Wiley \& Sons, Chichester.

Short A. 2006. Australian beach-systems-nature and distribution. Journal of Coastal Research 22(1): 11-27.

Short A \& C Hogan. 1994. Rip currents and beach hazards: Their impact on public safety and implications for coastal management. Journal of Coastal Research, Special Issue 12: Coastal Hazards: 197-209.

Trask P. 1932. Origin and environment of source sediments of petroleum, 323 pp. Gulf Publishing Company, Houston.

Wentworth C. 1922. A scale of grade and class terms for clastic sediments. Journal of Geology 30: 377-392.

Wright L \& A Short. 1984. Morphodynamic variability of surf zones and beaches: a synthesis. Marine Geology 56: 93-118.

Wright L, S May, A Short \& M Green. 1984. Beach and surf zone equilibria and response times. Proceedings of the $19^{\text {th }}$ Coastal Engineering Conference, pp. 2150-2164. American Society of Civil Engineers, Houston. 